\title{
Effects of Sex and Age on Quadriceps and Hamstring Strength and Flexibility in High School Basketball Athletes
}

\author{
Takashi Nagai $^{1}$ a , Nathaniel Bates ${ }^{2}$, April McPherson $^{3}$, Rena Hale ${ }^{2}$, Timothy Hewett ${ }^{4}$, Nathan D. Schilaty ${ }^{2}$ \\ 1 United States Army Research Institute of Environmental Medicine; Mayo Clinic, ${ }^{2}$ Mayo Clinic, ${ }^{3}$ United States Olympic \& Paralympic Committee, 4 \\ Rocky Mountain Consortium for Sports Research \\ Keywords: age, hamstring, high school basketball athletes, risk factors, sex
}

https://doi.org/10.26603/001c.27986

\section{International Journal of Sports Physical Therapy}

Vol. 16, Issue 5, 2021

\section{Background}

Eccentric hamstring strength and hamstring/quadriceps strength ratios have been identified as modifiable risk factors of hamstring strains. Additionally, those strength and flexibility characteristics are commonly used as clinical tests to monitor progress of athletes with acute or chronic hamstring strains. Although hamstring strains are common among basketball athletes, normative values of knee strength and flexibility characteristics are scarce. Normative values for these athletes would be important in prevention and management of hamstring strains.

\section{Purpose}

To establish quadriceps and hamstring isokinetic strength and flexibility values among high school basketball athletes and examine the effects of sex and age.

\section{Study Design}

Cross-sectional research

\section{Methods}

Isokinetic knee muscular strength (concentric quadriceps [QuadC], concentric hamstring [HamC], eccentric hamstring [HamE], and strength ratios ([HamC/QuadC and HamE/ Quad]), flexibility of hip flexors and quadriceps during a Modified Thomas test, and flexibility of hip extensors and hamstring during passive straight leg raise (SLR) and passive knee extension (PKE) tests were measured. Effects of sex and age were analyzed using t-tests and analysis of variance, respectively with Bonferroni corrected post hoc tests $(p \leqslant 0.01)$.

\section{Results}

A total of 172 high school basketball athletes (64 males/108 females; mean age (range): 15.7 (14-18) years old) participated in the study. Male athletes were significantly stronger than female athletes (QuadC: $p<0.001$; HamC: $p<0.001$ ) while no differences were observed in strength ratio (HamC/QuadC: $p=0.759-0.816$; HamE/QuadC: $p=0.022-0.061$ ). Among male athletes, a significant effect of age on quadriceps and hamstring strength was observed: older male athletes were stronger than younger male athletes. Contrarily, there were no effects of age on strength among female athletes. There were significant sex differences in quadriceps flexibility, SLR, and PKE (female athletes were more flexible; $p=0.001-0.005$ ) while no sex differences were found in hip flexor flexibility $(p=0.105-0.164)$. There were no effects of age for any flexibility variables within male and female athletes $(p=0.151-0.984)$.

\footnotetext{
a Corresponding Author:

Takashi Nagai, ATC, PhD

10 General Greene Avenue, Building 42, Natick, Massachusetts, USA 01760

508-206-2251

Fax: 508-206-2144

takashi.nagai.civ@mail.mil
} 


\section{Conclusion}

The current results provide normative values for hamstring strength and flexibility in high school basketball athletes. These normative values may further assist sports medicine specialists to develop screening tests, interventions, and return-to-sport criteria in this population.

\section{Level of Evidence}

3B

\section{INTRODUCTION}

Hamstring strains are significant and frequent musculoskeletal injuries as they typically result in persistent symptoms and lengthy recovery periods, which limit an athlete's ability to participate. ${ }^{1-4}$ Hamstring strains also exhibit high rates of re-injury. ${ }^{5,6}$ Professional and college basketball athletes are inherently at risk for hamstring strains as ballistic movements that include jumping and sprinting are common causes of hamstring strains. ${ }^{7-9}$ Based on the National Collegiate Athletic Association Injury Surveillance data, rate of hamstring strains in basketball was the sixth highest (after field hockey, soccer, outdoor track, lacrosse, and indoor track) among 13 women's sports while rate of hamstring strains in men's basketball was the third lowest among 12 men's sports. ${ }^{7}$ Additionally, rate of hamstring strains in female basketball athletes was twice as high as their male counterparts. ${ }^{7}$ Among high school basketball athletes, percentages of hip/thigh/upper leg injury rates (including hamstring strains, but, not specified) out of all basketball injuries were similar between sexes (girls: $8.7 \%$; boys: $8.2 \%$ of all injuries). ${ }^{10}$ Interestingly, these hip/thigh/ upper leg injuries occur more frequently during practices in girls and during games in boys. ${ }^{10}$ More epidemiological studies are needed to confirm sex differences in hamstring strains.

In an effort to reduce hamstring strains, there have been several prospective risk factors identified. Recent metaanalyses reveal that older age, higher body mass, higher body mass index, and a prior history of injury are prospective risk factors for lower extremity musculoskeletal injuries including hamstring strains. ${ }^{11,12}$ Among modifiable neuromuscular characteristics, lower quadriceps, hamstring, and hamstring-to-quadriceps strength ratios have been identified to increase prospective injury risk of hamstring strains in Australian football and soccer athletes. ${ }^{13-16}$ A lack of flexibility of the hip flexors discerned using the Modified Thomas test and of the hamstring using the straight-leg raise (SLR) test was identified prospectively to increase injury risk of hamstring strains among older ( $\geqslant 25$ years old) Australian football athletes ${ }^{17}$ and among male professional soccer athletes, ${ }^{18}$ respectively. Contrarily, several studies did not find these neuromuscular characteristics such as weaker eccentric hamstring strength ${ }^{19,20}$ and poor hamstring flexibility ${ }^{21}$ as prospective risk factors of hamstring strains. A systematic review has reported that concentric and eccentric hamstring muscular strength was not a strong prospective risk factor. ${ }^{22}$ Similarly, poor hamstring flexibility, using active knee extension (AKE), was not a prospective risk factor among Gaelic football athletes. ${ }^{21}$

From a rehabilitation perspective, hamstring eccentric strength (HamE) and the ratio of HamE divided by quadriceps concentric strength (QuadC) was significantly reduced in the injured limb compared to the contralateral uninjured limb in male athletes with hamstring strains. ${ }^{23}$ The authors utilized this particular ratio (HamE/QuadC) to screen athletes for risk of hamstring strains, to track rehabilitation progress, and to determine safe return-to-sport after hamstring strains. ${ }^{23,24}$ A systematic review also supports the contention that hamstring strength and flexibility testing could provide a valuable tool to monitor progress and safe return-to-sport after hamstring strains. ${ }^{25}$ This review also included average recovery time of hamstring strength (within 20 days) and flexibility (within 50 days) after hamstring strains. ${ }^{25}$ Although there are mixed findings on clinical importance of baseline isokinetic hamstring strength and flexibility testing, it appears beneficial in sports medicine / physical therapy clinics where each subject/patient has dedicated time and repeat testing to monitor progress objectively.

Although there is a high prevalence of hamstring strains in basketball (the second highest injury rates after indoor track and field among all indoor sports) and high clinical relevance for hamstring strength and flexibility testing, few studies have provided normative values for isokinetic hamstring strength and common clinical flexibility tests for each sex and age among high school basketball athletes. Generally, older male athletes exhibit stronger muscular strength $^{26}$ while female athletes are more flexible and exhibit better stretch pain tolerance. ${ }^{27}$ Understanding the effects of sex and age on these strength and flexibility characteristics is clinical relevant and important. Sports medicine specialists could quantitatively monitor athlete's progress and make a better decision on return-to-sport during rehabilitation. Therefore, the purpose of this study was to examine effects of age and sex on hamstring and quadriceps strength and flexibility in high school basketball athletes. The hypothesis was that male athletes and older athletes would exhibit significantly greater strength than female athletes and younger athletes, respectively. Contrarily, it was hypothesized that female athletes and younger athletes would exhibit significantly greater hamstring and hip flexors flexibility than male athletes and older athletes.

\section{METHODS}

The study was reviewed and approved by the Mayo Clinic Institutional Review Board (17-003905). This investigation was part of a large prospective study to examine the risk factors of hamstring strains. The current dataset was collected at the beginning of the high school basketball season in the first year of the project. Basketball athletes were 
recruited from local high schools and tested at the start of their basketball season. Informed consents and assents were obtained from each player and his/her parent (for 14-17 years old athletes). Inclusion criteria were ages 14-18 years old with no previous knee injuries or surgeries in the past year. Strength and flexibility testing each took approximately 15 minutes.

After height and weight were measured with a standard stadiometer and scale (Seca North America, East Hanover, $\mathrm{MD}$ ), knee extension (quadriceps concentric muscular strength) and knee flexion strength (hamstring concentric muscular strength) at $240^{\circ} / \mathrm{sec}$ and eccentric knee flexion strength (hamstring eccentric muscular strength) at $30^{\circ} / \mathrm{sec}$ were assessed using the HumacNorm dynamometer (CSMi, Stoughton, MA). These speeds for concentric and eccentric muscular strength testing were chosen based on the previous studies, examining risk factors of hamstring strains. ${ }^{23,24}$ Subjects were seated on the dynamometer chair with straps secured around shoulder, waist, and thigh to isolate movement to the targeted lower extremity during testing. The testing leg was securely attached to the dynamometer around the distal shank $3 \mathrm{~cm}$ proximal to the lateral malleolus with the axis of the dynamometer aligned with the knee axis of rotation. Then, limb weight was measured and accounted for each subject. After familiarization trials, subjects were asked to extend and flex their knee as hard as possible (and verbally encouraged) for a full-range of motion for 10 repetitions. Subjects then performed the hamstring eccentric strength test. Eccentric trials started with the knee at full flexion. Subjects were then asked to resist the dynamometer arm by flexing their knee as hard as possible while the dynamometer arm articulated their shank towards full extension. ${ }^{23}$ Peak knee flexion torque was recorded during three trials. This methodology has previously reported good reliability. ${ }^{28}$ The current authors have also established good test-retest reliability of 20 participants using the same testing protocols and found intraclass correlation coefficient ranges from 0.706 to 0.937 . For statistical analyses, the average of 10 and three trials were used for concentric and eccentric strength, respectively. The average peak torque $(\mathrm{Nm})$ normalized to body mass $(\mathrm{kg})$ was used for analyses (\%BM). The concentric hamstring over concentric quadriceps strength ratio (HamC/QuadC) was calculated by the peak hamstring concentric strength divided by the peak quadriceps concentric strength multiplied by 100 in order to express a percentage. A ratio of the peak hamstring eccentric strength over the peak quadriceps concentric strength (HamE/QuadC) was also use for statistical analyses.

A Modified Thomas test was used to assess flexibility of the iliopsoas and quadriceps muscles. Subjects laid in a supine position with the ischial tuberosities on the edge of treatment table. A clinician elevated both subject's legs simultaneously into hip flexion until a neutral pelvis position was established. From this position, the clinician held the contralateral leg stable and slowly lowered the testing leg. The assistant placed a digital inclinometer (Johnson Level \& Tool, Mequon, WI) on top of the midpoint of the thigh and shin to measure the iliopsoas and quadriceps flexibility, respectively. The inclinometer was initially calibrated to 0 degrees on the horizon, and higher values represent greater iliopsoas and quadriceps flexibility.

Flexibility of the posterior hip (gluteal and hamstring muscles) and posterior knee (hamstring muscles) was assessed using passive SLR and passive knee extension (PKE), respectively. ${ }^{29}$ For the passive SLR, subjects laid supine while a clinician flexed their hip and kept their leg straight. Each subject's pelvis was stabilized with a belt. An inclinometer was placed on the top of the shin midpoint. Flexibility was measured from the horizontal position (higher values represented greater flexibility). PKE was performed in a supine position with the testing leg positioned at $90 \mathrm{de}-$ grees hip and knee flexion. ${ }^{30}$ The examiner stabilized the testing leg's thigh vertically and passively moved the lower leg to the point of muscle tautness. An inclinometer was placed on the top of the shin midpoint to measure flexibility (higher values represented greater flexibility). This procedure was repeated a few times until the examiner found a consistent end point of muscle tautness, and the last value was taken for statistical analysis.

Descriptive statistics (means and standard deviations) were calculated in each sex and age group. Each dependent variable was screened for normality using Shapiro-Wilk tests. Given the wide range of ages, sex differences were examine using independent t-tests or Mann-Whitney tests for each age group. Effects of age within male and female athletes were examined using one-way between-subjects analysis of variance (ANOVA) or Kruskal Wallis tests with five age-levels $(14,15,16,17$, and 18 years old). In addition to normality, homogeneity of variance assumption was screened using Levene's tests. If ANOVA/Kruskal Wallis tests were significant, post-hoc analyses were used to compare between each age group. In order to reduce Type $1 \mathrm{Er}-$ ror due to multiple comparisons, significance was adjusted with Bonferroni and set at $p \leqslant 0.01$ a priori.

\section{RESULTS}

A total of 64 males ( 14 years old: $n=8 ; 15$ years old: $n=17 ; 16$ years old: $n=19 ; 17$ years old: $n=13 ; 18$ years old: $n=7)$ and 108 females $(14$ years old: $n=27 ; 15$ years old: $n=33 ; 16$ years old: $n=15 ; 17$ years old: $n=23 ; 18$ years old: $n=10$ ) participated in the study. Demographics for male and female athletes as well as their age stratification are in Table 1.

For strength and strength ratio variables, descriptive statistics are shown in Table $2 \mathrm{a}-\mathrm{c}$ and $\underline{\mathrm{3} a-b}$, respectively. Overall, there were significant sex differences in quadriceps concentric and hamstring concentric strength $(p<0.001)$. Specifically, sex differences in quadriceps and hamstring concentric strength were observed among the older ages: 16 years old $(p<0.005), 17$ years old $(p=0.003)$ and 18 years old $(p=0.001)$. Hamstring eccentric strength $(p=0.024)$ and strength ratios $(p=0.022-0.816)$ were not statistically different between sexes.

There were significant effects of age on all strength variables within male athletes $(p=0.001-0.031)$. Specifically, quadriceps and hamstring strength values were lower in 14 and 15 years old male athletes than 16 and 18 years old male athletes. Contrarily, female athletes did not show any effects of age on strength variables ( $p=0.048-0.856$ ).

Flexibility variables are shown in Table $4 a-b$ and $\underline{5 a-b}$. Iliopsoas (hip flexors) and quadriceps flexibility (Modified 
Table 1. Subject Demographics

\begin{tabular}{ccccccc}
\hline & \multicolumn{2}{c}{ Height $(\mathrm{cm})$} & Sex Diff & \multicolumn{2}{c}{ Weight (kg) } & Sex Diff \\
\hline Age & Males & Females & $p$-value & Males & Females & $p$-value \\
\hline 14 & $172.9 \pm 6.3$ & $168.4 \pm 9.7$ & 0.237 & $69.6 \pm 9.1$ & $63.2 \pm 14.8$ & 0.258 \\
15 & $178.2 \pm 7.5$ & $169.9 \pm 8.4$ & $0.001^{*}$ & $66.7 \pm 12.4$ & $66.6 \pm 14.7$ & 0.814 \\
16 & $182.6 \pm 9.7$ & $168.5 \pm 3.9$ & $<0.001^{*}$ & $73.8 \pm 10.6$ & $67.6 \pm 8.4$ & 0.137 \\
17 & $184.4 \pm 8.5$ & $173.5 \pm 8.2$ & $0.001^{*}$ & $78.9 \pm 12.4$ & $69.0 \pm 16.1$ & 0.031 \\
18 & $184.0 \pm 2.7$ & $170.9 \pm 10.0$ & $0.002^{*}$ & $80.9 \pm 8.2$ & $66.9 \pm 7.9$ & $0.003^{*}$ \\
All & $180.7 \pm 8.6$ & $170.2 \pm 8.5$ & $<0.001^{*}$ & $73.2 \pm 11.9$ & $66.4 \pm 13.8$ & $<0.001^{*}$ \\
\hline $\begin{array}{c}\text { Age Diff } \\
p \text {-value }\end{array}$ & 0.011 & 0.250 & & 0.015 & 0.553 &
\end{tabular}

* represents significant differences between sexes $(p \leqslant 0.01)$.

Table 2a. Effects of sex and age on quadriceps concentric strength (QuadC)

\begin{tabular}{|c|c|c|c|c|c|c|}
\hline \multirow[b]{2}{*}{ Age } & \multicolumn{2}{|c|}{ Left Leg (\%BM) } & \multirow{2}{*}{$\begin{array}{l}\text { Sex Diff } \\
p \text {-value }\end{array}$} & \multicolumn{2}{|c|}{ Right Leg (\%BM) } & \multirow{2}{*}{$\begin{array}{l}\text { Sex Diff } \\
p \text {-value }\end{array}$} \\
\hline & Males & Females & & Males & Females & \\
\hline 14 & $125.5 \pm 30.4$ & $112.1 \pm 19.6$ & 0.167 & $125.5 \pm 34.8$ & $114.1 \pm 23.2$ & 0.314 \\
\hline 15 & $141.1 \pm 33.0$ & $121.9 \pm 16.6$ & 0.071 & $139.8 \pm 25.9$ & $122.4 \pm 21.1$ & 0.032 \\
\hline 16 & $162.7 \pm 22.9$ & $110.1 \pm 22.3$ & $<0.001^{*}$ & $161.6 \pm 28.2$ & $114.3 \pm 19.3$ & $<0.001^{*}$ \\
\hline 17 & $141.5 \pm 11.3$ & $125.0 \pm 21.6$ & $0.003^{*}$ & $151.5 \pm 30.4$ & $123.3 \pm 21.7$ & $0.003^{*}$ \\
\hline 18 & $162.0 \pm 30.6$ & $128.5 \pm 26.6$ & 0.034 & $161.3 \pm 23.5$ & $120.4 \pm 41.2$ & 0.034 \\
\hline All & $147.8 \pm 28.4$ & $118.8 \pm 21.1$ & $<0.001^{*}$ & $149.2 \pm 30.1$ & $119.1 \pm 23.8$ & $<0.001^{*}$ \\
\hline $\begin{array}{l}\text { Age Diff } \\
p \text {-value }\end{array}$ & $\begin{array}{c}0.004 \# ; \\
14,15,17<16\end{array}$ & 0.048 & & 0.031 & 0.580 & \\
\hline
\end{tabular}

* represents significant differences between sexes $(p \leqslant 0.01)$. \# represents significant differences among ages $(p \leqslant 0.01)$.

Table 2b. Effects of sex and age on hamstring concentric strength (HamC)

\begin{tabular}{|c|c|c|c|c|c|c|}
\hline \multirow[b]{2}{*}{ Age } & \multicolumn{2}{|c|}{ Left Leg (\%BM) } & \multirow{2}{*}{$\begin{array}{l}\text { Sex Diff } \\
p \text {-value }\end{array}$} & \multicolumn{2}{|c|}{ Right Leg (\%BM) } & \multirow{2}{*}{$\begin{array}{l}\text { Sex Diff } \\
p \text {-value }\end{array}$} \\
\hline & Males & Females & & Males & Females & \\
\hline 14 & $61.9 \pm 13.8$ & $55.2 \pm 12.0$ & 0.281 & $56.7 \pm 20.4$ & $53.9 \pm 14.0$ & 0.676 \\
\hline 15 & $62.3 \pm 15.3$ & $56.9 \pm 17.4$ & 0.302 & $65.9 \pm 15.9$ & $55.4 \pm 17.4$ & 0.053 \\
\hline 16 & $77.8 \pm 15.8$ & $53.6 \pm 12.8$ & $<0.001^{*}$ & $75.1 \pm 17.8$ & $59.3 \pm 13.4$ & $0.009^{*}$ \\
\hline 17 & $68.7 \pm 15.1$ & $59.0 \pm 12.4$ & 0.048 & $64.4 \pm 12.0$ & $60.5 \pm 16.1$ & 0.465 \\
\hline 18 & $90.3 \pm 15.8$ & $63.0 \pm 13.4$ & $0.002^{*}$ & $91.3 \pm 16.2$ & $58.9 \pm 12.1$ & $<0.001^{*}$ \\
\hline All & $71.2 \pm 17.5$ & $57.0 \pm 13.9$ & $<0.001^{*}$ & $70.0 \pm 18.5$ & $57.1 \pm 15.2$ & $<0.001^{*}$ \\
\hline $\begin{array}{l}\text { Age Diff } \\
p \text {-value }\end{array}$ & $\begin{array}{c}0.001 \# ; \\
14,15<18\end{array}$ & 0.276 & & $\begin{array}{c}0.001 \# ; \\
14,15,17<18\end{array}$ & 0.480 & \\
\hline
\end{tabular}

* represents significant differences between sexes $(p \leqslant 0.01)$. \# represents significant differences among ages $(p \leqslant 0.01)$.

Thomas test) were not significantly different between sexes $(p=0.015-0.164)$ except the quadriceps flexibility of the left limb (males: 81.1 degrees, females: 84.0 degrees, $p=0.005$ ). For SLR and PKE tests, female athletes exhibited significantly greater hamstring flexibility $(p<0.001)$. Among all flexibility variables, there were no significant effects of age $(p=0.151-0.984)$.

\section{DISCUSSION}

The current study examined sex and age differences in hamstring and quadriceps muscular strength and flexibility. For strength variables, the hypothesis was partially supported as sex differences in both hamstring and quadriceps 
Table 2c. Effects of sex and age on hamstring eccentric strength (HamE)

\begin{tabular}{|c|c|c|c|c|c|c|}
\hline \multirow[b]{2}{*}{ Age } & \multicolumn{2}{|c|}{ Left Leg (\%BW) } & \multirow{2}{*}{$\begin{array}{l}\text { Sex Diff } \\
p \text {-value }\end{array}$} & \multicolumn{2}{|c|}{ Right Leg (\%BW) } & \multirow{2}{*}{$\begin{array}{l}\text { Sex Diff } \\
p \text {-value }\end{array}$} \\
\hline & Males & Females & & Males & Females & \\
\hline 14 & $145.7 \pm 39.0$ & $162.7 \pm 47.1$ & 0.390 & $162.6 \pm 44.9$ & $167.1 \pm 51.2$ & 0.834 \\
\hline 15 & $166.8 \pm 42.1$ & $178.4 \pm 45.7$ & 0.409 & $156.4 \pm 36.4$ & $177.2 \pm 48.0$ & 0.196 \\
\hline 16 & $219.3 \pm 65.1$ & $156.4 \pm 48.8$ & $0.005^{*}$ & $214.3 \pm 50.8$ & $160.3 \pm 50.3$ & $0.005^{*}$ \\
\hline 17 & $204.4 \pm 61.1$ & $175.2 \pm 50.9$ & 0.138 & $204.1 \pm 77.7$ & $176.3 \pm 49.3$ & 0.302 \\
\hline 18 & $229.1 \pm 56.0$ & $171.9 \pm 41.0$ & 0.033 & $218.5 \pm 41.4$ & $167.9 \pm 51.0$ & 0.051 \\
\hline All & $194.2 \pm 60.4$ & $169.5 \pm 47.1$ & 0.024 & $190.5 \pm 57.7$ & $170.9 \pm 49.1$ & 0.024 \\
\hline $\begin{array}{l}\text { Age Diff } \\
p \text {-value }\end{array}$ & $0.005 \# ; 14<16$ & 0.579 & & $0.006 \# ; 15<16,18$ & 0.856 & \\
\hline
\end{tabular}

* represents significant differences between sexes $(p \leqslant 0.01)$. \# represents significant differences among ages $(p \leqslant 0.01)$.

Table 3a. Effects of sex and age on concentric hamstring / quadriceps strength ratios (HamC/QuadC)

\begin{tabular}{|c|c|c|c|c|c|c|}
\hline \multirow[b]{2}{*}{ Age } & \multicolumn{2}{|c|}{ Left Leg (\%) } & \multirow{2}{*}{$\begin{array}{l}\text { Sex Diff } \\
p \text {-value }\end{array}$} & \multicolumn{2}{|c|}{ Right Leg (\%) } & \multirow{2}{*}{$\begin{array}{l}\text { Sex Diff } \\
p \text {-value }\end{array}$} \\
\hline & Males & Females & & Males & Females & \\
\hline 14 & $51.4 \pm 14.0$ & $50.0 \pm 10.2$ & 0.762 & $48.8 \pm 20.3$ & $48.7 \pm 14.4$ & 0.995 \\
\hline 15 & $44.8 \pm 10.4$ & $47.4 \pm 15.3$ & 0.540 & $47.3 \pm 9.0$ & $46.4 \pm 15.3$ & 0.804 \\
\hline 16 & $48.3 \pm 9.6$ & $51.1 \pm 17.8$ & 0.573 & $47.7 \pm 13.9$ & $52.9 \pm 13.0$ & 0.289 \\
\hline 17 & $48.6 \pm 9.9$ & $48.2 \pm 12.0$ & 0.933 & $43.4 \pm 8.1$ & $49.6 \pm 13.1$ & 0.131 \\
\hline 18 & $56.3 \pm 8.2$ & $50.5 \pm 12.7$ & 0.311 & $57.5 \pm 13.0$ & $44.5 \pm 8.7$ & 0.038 \\
\hline All & $48.7 \pm 10.6$ & $49.1 \pm 13.4$ & 0.816 & $47.9 \pm 12.6$ & $48.6 \pm 13.7$ & 0.759 \\
\hline $\begin{array}{l}\text { Age Diff } \\
p \text {-value }\end{array}$ & 0.148 & 0.874 & & 0.213 & 0.572 & \\
\hline
\end{tabular}

Table 3b. Effects of sex and age on eccentric hamstring / concentric quadriceps strength ratios (HamE/QuadC)

\begin{tabular}{|c|c|c|c|c|c|c|}
\hline \multirow[b]{2}{*}{ Age } & \multicolumn{2}{|c|}{ Left Leg (\%) } & \multirow{2}{*}{$\begin{array}{l}\text { Sex Diff } \\
p \text {-value }\end{array}$} & \multicolumn{2}{|c|}{ Right Leg (\%) } & \multirow{2}{*}{$\begin{array}{l}\text { Sex Diff } \\
p \text {-value }\end{array}$} \\
\hline & Males & Females & & Males & Females & \\
\hline 14 & $118.2 \pm 27.1$ & $145.7 \pm 35.5$ & 0.069 & $136.1 \pm 40.3$ & $147.0 \pm 36.0$ & 0.496 \\
\hline 15 & $119.7 \pm 25.3$ & $148.0 \pm 41.0$ & 0.009* & $114.3 \pm 31.1$ & $146.4 \pm 38.0$ & $0.008^{*}$ \\
\hline 16 & $134.6 \pm 34.3$ & $147.2 \pm 52.8$ & 0.425 & $134.7 \pm 32.2$ & $140.5 \pm 40.7$ & 0.882 \\
\hline 17 & $143.7 \pm 37.6$ & $141.7 \pm 38.6$ & 0.878 & $132.7 \pm 30.8$ & $144.8 \pm 40.6$ & 0.361 \\
\hline 18 & $141.6 \pm 23.3$ & $141.7 \pm 57.5$ & 0.998 & $135.4 \pm 17.0$ & $136.0 \pm 42.9$ & 0.972 \\
\hline All & $131.3 \pm 31.6$ & $145.2 \pm 42.1$ & 0.061 & $128.8 \pm 31.6$ & $144.4 \pm 38.2$ & 0.022 \\
\hline $\begin{array}{c}\text { Age Diff } \\
p \text {-value }\end{array}$ & 0.169 & 0.881 & & 0.290 & 0.880 & \\
\hline
\end{tabular}

* represents significant differences between sexes $(p \leqslant 0.01)$.

strength were observed except for eccentric strength. All isokinetic strength values in the current study were similar in magnitude to the previous studies in high school athletes. ${ }^{26,31,32}$ It is interesting to note that sex differences become more apparent in later ages as male athletes become stronger while female athletes exhibit similar strength levels across ages in high school. The current findings align with the previous study that identifies stronger quadriceps and hamstring strength in males and an absence of strength changes in female athletes in middle/high school. ${ }^{26}$

The current results provide normative values for hamstring strength and flexibility in high school basketball athletes. Since there are few normative values available in the literature, these results are clinically important for physical therapists, athletic trainers, or other clinicians who regularly utilize an isokinetic dynamometer. When combined 
Table 4a. Effects of sex and age on hip flexors flexibility during Modified Thomas test

\begin{tabular}{|c|c|c|c|c|c|c|}
\hline \multirow[b]{2}{*}{ Age } & \multicolumn{2}{|c|}{ Left Leg (degrees) } & \multirow{2}{*}{$\begin{array}{l}\text { Sex Diff } \\
p \text {-value }\end{array}$} & \multicolumn{2}{|c|}{ Right Leg (degrees) } & \multirow{2}{*}{$\begin{array}{l}\text { Sex Diff } \\
p \text {-value }\end{array}$} \\
\hline & Males & Females & & Males & Females & \\
\hline 14 & $4.5 \pm 2.4$ & $4.7 \pm 3.9$ & 0.923 & $5.0 \pm 1.8$ & $5.1 \pm 4.2$ & 0.576 \\
\hline 15 & $4.0 \pm 3.9$ & $4.2 \pm 5.1$ & 0.829 & $4.2 \pm 5.4$ & $4.9 \pm 5.5$ & 0.459 \\
\hline 16 & $2.8 \pm 3.9$ & $6.7 \pm 3.0$ & $0.003^{*}$ & $4.5 \pm 6.2$ & $7.9 \pm 5.0$ & 0.091 \\
\hline 17 & $5.9 \pm 12.7$ & $5.0 \pm 3.4$ & 0.721 & $6.5 \pm 9.7$ & $6.0 \pm 5.7$ & 0.603 \\
\hline 18 & $4.4 \pm 4.2$ & $3.7 \pm 2.3$ & 0.653 & $5.1 \pm 4.4$ & $5.8 \pm 6.2$ & 0.813 \\
\hline All & $4.2 \pm 6.5$ & $4.8 \pm 4.0$ & 0.105 & $4.9 \pm 6.3$ & $5.7 \pm 5.2$ & 0.164 \\
\hline $\begin{array}{c}\text { Age Diff } \\
p \text {-value }\end{array}$ & 0.394 & 0.151 & & 0.956 & 0.393 & \\
\hline
\end{tabular}

* represents significant differences between sexes $(p \leqslant 0.01)$.

Table 4b. Effects of sex and age on quadriceps flexibility during Modified Thomas test

\begin{tabular}{|c|c|c|c|c|c|c|}
\hline & \multicolumn{2}{|c|}{ Left Leg (degrees) } & \multirow{2}{*}{$\begin{array}{c}\text { Sex Diff } \\
p \text {-value }\end{array}$} & \multicolumn{2}{|c|}{ Right Leg (degrees) } & \multirow{2}{*}{$\begin{array}{l}\text { Sex Diff } \\
p \text {-value }\end{array}$} \\
\hline Age & Males & Females & & Males & Females & \\
\hline 14 & $83.9 \pm 4.2$ & $84.6 \pm 5.8$ & 0.576 & $81.4 \pm 4.4$ & $82.6 \pm 5.8$ & 0.363 \\
\hline 15 & $82.3 \pm 4.6$ & $84.6 \pm 4.4$ & 0.063 & $78.9 \pm 8.4$ & $82.3 \pm 5.6$ & 0.093 \\
\hline 16 & $81.6 \pm 6.8$ & $82.6 \pm 5.8$ & 0.636 & $80.6 \pm 8.3$ & $79.9 \pm 7.4$ & 0.789 \\
\hline 17 & $78.7 \pm 9.8$ & $84.6 \pm 5.3$ & 0.043 & $73.2 \pm 21.2$ & $83.8 \pm 4.8$ & $0.006^{*}$ \\
\hline 18 & $78.6 \pm 9.5$ & $81.5 \pm 8.1$ & 0.417 & $81.3 \pm 6.1$ & $80.0 \pm 7.2$ & 0.707 \\
\hline All & $81.1 \pm 7.1$ & $84.0 \pm 5.5$ & $0.005^{*}$ & $78.8 \pm 11.7$ & $82.2 \pm 6.0$ & 0.015 \\
\hline $\begin{array}{l}\text { Age Diff } \\
p \text {-value }\end{array}$ & 0.392 & 0.539 & & 0.651 & 0.372 & \\
\hline
\end{tabular}

* represents significant differences between sexes $(p \leqslant 0.01)$.

Table 5a. Effects of sex and age on posterior thigh and hip flexibility during passive straight leg raise (SLR) test

\begin{tabular}{|c|c|c|c|c|c|c|}
\hline \multirow[b]{2}{*}{ Age } & \multicolumn{2}{|c|}{ SLR (degrees) Left } & \multirow{2}{*}{$\begin{array}{c}\text { Sex Diff } \\
p \text {-value }\end{array}$} & \multicolumn{2}{|c|}{ SLR (degrees) Right } & \multirow{2}{*}{$\begin{array}{l}\text { Sex Diff } \\
p \text {-value }\end{array}$} \\
\hline & Males & Females & & Males & Females & \\
\hline 14 & $61.4 \pm 6.1$ & $74.9 \pm 8.2$ & $<0.001^{*}$ & $64.3 \pm 6.1$ & $75.5 \pm 8.6$ & $0.002^{*}$ \\
\hline 15 & $66.9 \pm 8.5$ & $75.2 \pm 10.7$ & $0.007^{*}$ & $68.1 \pm 8.2$ & $74.9 \pm 9.0$ & 0.013 \\
\hline 16 & $66.0 \pm 7.6$ & $74.7 \pm 9.0$ & $0.004^{*}$ & $67.9 \pm 9.8$ & $74.7 \pm 7.0$ & 0.029 \\
\hline 17 & $61.9 \pm 10.5$ & $75.9 \pm 10.2$ & $<0.001^{*}$ & $62.6 \pm 10.1$ & $76.6 \pm 10.2$ & $<0.001^{*}$ \\
\hline 18 & $65.0 \pm 5.6$ & $79.1 \pm 6.2$ & $<0.001^{*}$ & $64.6 \pm 6.3$ & $78.2 \pm 7.1$ & $0.001^{*}$ \\
\hline All & $64.7 \pm 8.2$ & $75.6 \pm 9.3$ & $<0.001^{*}$ & $66.1 \pm 8.8$ & $75.7 \pm 8.7$ & $<0.001^{*}$ \\
\hline $\begin{array}{l}\text { Age Diff } \\
p \text {-value }\end{array}$ & 0.349 & 0.776 & & 0.372 & 0.817 & \\
\hline
\end{tabular}

* represents significant differences between sexes $(\mathrm{p} \leqslant 0.01)$.

with the previous findings (recovery time of hamstring strength within 20 days \& flexibility within 50 days after hamstring strains) ${ }^{25}$, clinicians can utilize isokinetic hamstring strength and flexibility tests to objectively monitor rehabilitation progress and to determine the timing of return-to-sport.

When strength ratios were examined, no significant sex differences were observed, which is contrary to previous literature. ${ }^{33}$ Therefore, the hypothesis that males would have higher strength ratios than females was rejected. A concept of hamstring eccentric strength over quadriceps concentric strength ratio was developed two decades ago ${ }^{34}$ and has been utilized to screen athletes. ${ }^{23,24}$ Compared to the reported ratio (50\%-60\%), the current HamC/QuadC values 
Table 5b. Effects of sex and age on posterior thigh and hip flexibility during passive knee extension (PKE) test

\begin{tabular}{|c|c|c|c|c|c|c|}
\hline \multirow[b]{2}{*}{ Age } & \multicolumn{2}{|c|}{ Left Leg (degrees) } & \multirow{2}{*}{$\begin{array}{l}\text { Sex Diff } \\
p \text {-value }\end{array}$} & \multicolumn{2}{|c|}{ Right Leg (degrees) } & \multirow{2}{*}{$\begin{array}{l}\text { Sex Diff } \\
p \text {-value }\end{array}$} \\
\hline & Males & Females & & Males & Females & \\
\hline 14 & $52.1 \pm 10.4$ & $58.7 \pm 10.1$ & 0.116 & $53.4 \pm 10.2$ & $62.3 \pm 9.1$ & 0.023 \\
\hline 15 & $50.0 \pm 9.1$ & $57.1 \pm 10.7$ & 0.023 & $53.0 \pm 8.1$ & $61.6 \pm 8.8$ & $0.001^{*}$ \\
\hline 16 & $52.2 \pm 9.3$ & $57.5 \pm 7.5$ & 0.080 & $53.7 \pm 7.5$ & $61.7 \pm 6.9$ & $0.003^{*}$ \\
\hline 17 & $49.7 \pm 10.8$ & $60.0 \pm 9.2$ & $0.005^{*}$ & $51.3 \pm 8.1$ & $63.0 \pm 9.3$ & $0.001^{*}$ \\
\hline 18 & $48.9 \pm 8.0$ & $58.3 \pm 9.7$ & 0.051 & $51.3 \pm 4.5$ & $62.1 \pm 10.6$ & 0.013 \\
\hline All & $50.7 \pm 9.4$ & $58.3 \pm 9.6$ & $<0.001^{*}$ & $52.7 \pm 7.7$ & $62.1 \pm 8.8$ & $<0.001^{*}$ \\
\hline $\begin{array}{l}\text { Age Diff } \\
p \text {-value }\end{array}$ & 0.890 & 0.864 & & 0.912 & 0.984 & \\
\hline
\end{tabular}

* represents significant differences between sexes $(\mathrm{p} \leqslant 0.01)$.

were similar (43\%-56\%); however, the HamE/QuadC ratio in the current study (114\%-148\%) was higher than the previous study (75\%-90\%). ${ }^{23}$ Likely, differences in demographics such as age (25 years old vs. 14-18 years old in the current study) and testing procedures and methodologies potentially contribute to the current results.

Higher HamE/QuadC ratio was observed in female athletes. There are two potential reasons. First, female athletes exhibited a larger sex difference in quadriceps strength (females being $20-26 \%$ weaker) than a sex difference in the hamstring eccentric strength (females being 10-13\% weaker), which naturally inflated the strength ratio. Second, most athletes exhibited greater variability during eccentric strength testing than during concentric strength testing. The overall coefficient of variation (a measurement of relative variability defined as the ratio of the standard deviation divided by the mean ${ }^{35}$ ) during hamstring eccentric strength testing was $30-31 \%$ and $27-28 \%$ in males and females, respectively. As a comparison, the coefficient of variation during quadriceps and hamstring concentric strength were $20 \%$ and $25-26 \%$ in male athletes and $18-20 \%$ and $24-27 \%$ in female athletes, respectively. Most likely, this protocol was the first time that these athletes had engaged in eccentric strength testing. Although test-retest reliability was good to excellent, high coefficient of variation suggests that peak torque values can fluctuate among three trials. Additional familiarization and practice trials during eccentric hamstring muscular strength testing might have produced more consistent values.

Muscular strength weakness and lower hamstring/ quadriceps strength ratio have been identified as prospective risk factors in Australian-rule football and soccer athletes. ${ }^{24,36}$ Although the current subjects were younger than the subjects in those studies, ${ }^{24,36}$ lower strength ratio may play an important role in screening of individuals for a prospective risk factor of hamstring strains. In fact, a preliminary analysis of three female athletes who later suffered hamstring strains (within a few months after the baseline testing) demonstrated that they exhibited weaker hamstring eccentric strength (mean for injured athletes: 137.2\%BM vs. mean for non-injured athletes: $170.2 \% \mathrm{BM}$ ) and lower HamE/QuadC ratio (mean for injured athletes: $112.3 \%$ vs. mean for non-injured $145.5 \%$ ) when compared to the baseline values of female athletes who did not go on to injury. More injured subjects would be needed to run statistical analyses to explore this preliminary observation.

The current study was not aimed to establish the association between baseline strength and prospective hamstring strains. Instead, it aimed to establish normative values for hamstring strength and flexibility characteristics among high school basketball athletes. Regardless of athletes' background and competition levels, hamstring exercises could reduce hamstring strains by $50 \%$ on average. $37-39$ Sports medicine specialists and coaches should incorporate hamstring exercises gradually for high school basketball athletes for their athletic performance development as well as hamstring strain injury prevention purposes with proper progressions. ${ }^{40}$ Eccentric exercises have been commonly used during rehabilitation of tendinopathies, muscle strains, and anterior cruciate ligament post-operation. ${ }^{41}$ Future studies can focus on eccentric exercise dose-response relationship and establish return-to-sport criteria for athletes with acute or chronic hamstring strains.

For flexibility variables, female athletes exhibited greater flexibility in both SLR and PKE tests. However, there were no effect of sex and age on hip flexors and quadriceps flexibility during the Modified Thomas test. From a clinical perspective, the SLR is used to target/stretch the proximal hamstring and hip extensors while PKE is used to target/ stretch the extensibility of the distal hamstring with less constraint of the more proximal hip extensors. ${ }^{29}$ Based on the current findings, both flexibility tests for hamstring were sensitive enough to detect sex differences in high school basketball athletes. The current findings agree with previous studies. ${ }^{27,42}$

The current data also indicated that there were no significant effects of age on flexibility. That is also in accordance with a previous study. ${ }^{42}$ Interestingly, hamstring flexibility does not seem to change much throughout the lifespan from 20-29 years old group to 70-79 years old group. ${ }^{42}$ For younger populations, sex differences in general tissue laxity, commonly assessed with the Beighton Joint Mobility Test, ${ }^{43}$ may occur at the onset of puberty (ages: $11-12$ years on both sexes). ${ }^{43}$ Therefore, the youngest group (14 years old) in the current study had likely entered the pubertal stage of their maturation. Nonetheless, the current findings 
with high school basketball athletes with ages $14-18$ years adds to the existing literature and confirms that there are no age-related changes in hamstring flexibility among high school basketball athletes.

Decreased hamstring flexibility has identified as a risk factor for hamstring strains among male Australian-rule footballers and male professional soccer athletes. ${ }^{17,18,44}$ Since stretching exercises are already a part of most warmup and cool-down exercises, few clinical studies have been conducted to actually evaluate the effects of stretching on hamstring strains. ${ }^{45}$ Based on the current findings and available literature, male athletes may benefit from a stretching program to a larger extent than female athletes who already have greater flexibility.

The authors recognize limitations in this study. First, as a limitation of the research design (cross-sectional study and age-/sex-group comparisons), the current results cannot be interpreted as an absence of strength changes. In a longitudinal study over three years (from 11 years to 14 years old), knee extension strength increased in girls. ${ }^{46}$ Therefore, the current findings should be interpreted cautiously and used to reflect sex- and age-group differences in their strength and flexibility. Second, because the current study used age as one of the inclusion criteria, the stages of biological maturation were not evaluated. Stratification by maturation scales such as Pubertal Maturation Observational Scale (PMOS) ${ }^{47,48}$ or Tanner Stage ${ }^{49}$ might be helpful with the youth population. Therefore, the current results only represent the age effect in high school basketball athletes. The authors are aware that the current research design is a cross-sectional study; therefore, the association between strength/flexibility and injury risk of hamstring strains cannot be established.

\section{CONCLUSIONS}

The results of this study indicate that male high school basketball athletes were stronger and less flexible than female athletes while younger male athletes were weaker than older males. The same trend was not found in female athletes. Future studies should explore if any of these differences might be associated with athletes with hamstring strains. The current results expand the available evidence on hamstring strength and flexibility characteristics in high school basketball athletes. These normative values may further assist sports medicine specialists to develop screening tests, interventions, and return-to-sport criteria in this population.

\section{CONFLICTS OF INTEREST}

No authors have any conflict of interests to disclose.

\section{ACKNOWLEDGEMENTS}

Funding from the NBA \& GE Healthcare Orthopedics and Sports Medicine Collaboration, and NIH grant funding R01AR056259, R01AR055563, K12HD065987, L30AR070273 and T32AR056950.

\section{DISCLAIMER}

The views expressed in this manuscript are those of the authors and do not reflect the official policy of the Department of Army, Department of Defense, or the U.S. Government.

Submitted: October 30, 2020 CDT, Accepted: June 15, 2021 CDT 


\section{REFERENCES}

1. Ahmad CS, Redler LH, Ciccotti MG, Maffulli N, Longo UG, Bradley J. Evaluation and management of hamstring injuries. Am J Sports Med. 2013;41(12):2933-2947. doi:10.1177/03635465134870 $\underline{63}$

2. Ali K, Leland JM. Hamstring strains and tears in the athlete. Clin Sports Med. 2012;31(2):263-272. doi:10.1 016/j.csm.2011.11.001

3. Clanton TO, Coupe KJ. Hamstring strains in athletes: diagnosis and treatment. J Am Acad Orthop Surg. 1998;6(4):237-248.

4. Cohen S, Bradley J. Acute proximal hamstring rupture. J Am Acad Orthop Surg. 2007;15(6):350-355.

5. Warren P, Gabbe BJ, Schneider-Kolsky M, Bennell KL. Clinical predictors of time to return to competition and of recurrence following hamstring strain in elite Australian footballers. Br J Sports Med. 2010;44(6):415-419. doi:10.1136/bjsm.2008.048181

6. Heiderscheit BC, Sherry MA, Silder A, Chumanov ES, Thelen DG. Hamstring strain injuries: recommendations for diagnosis, rehabilitation, and injury prevention. J Orthop Sports Phys Ther. 2010;40(2):67-81. doi:10.2519/jospt.2010.3047

7. Dalton SL, Kerr ZY, Dompier TP. Epidemiology of hamstring strains in 25 NCAA sports in the 2009-2010 to 2013-2014 academic years. Am J Sports Med. 2015;43(11):2671-2679. doi:10.1177/03635465155996 $\underline{31}$

8. Deitch JR, Starkey C, Walters SL, Moseley JB. Injury risk in professional basketball players: a comparison of Women's National Basketball Association and National Basketball Association athletes. Am J Sports Med. 2006;34(7):1077-1083.

9. Drakos MC, Domb B, Starkey C, Callahan L, Allen AA. Injury in the national basketball association: a 17-year overview. Sports Health. 2010;2(4):284-290. $\underline{\mathrm{d}}$ oi:10.1177/1941738109357303

10. Borowski LA, Yard EE, Fields SK, Comstock RD. The epidemiology of US high school basketball injuries, 2005-2007. Am J Sports Med. 2008;36(12):2328-2335. doi:10.1177/03635465083228 $\underline{93}$
11. Green B, Bourne MN, van Dyk N, Pizzari T. Recalibrating the risk of hamstring strain injury (HSI): A 2020 systematic review and meta-analysis of risk factors for index and recurrent hamstring strain injury in sport. Br J Sports Med. 2020;54(18):1081-1088. doi:10.1136/bjsports-2019-10 $\underline{0983}$

12. Collings TJ, Bourne MN, Barrett RS, du Moulin W, Hickey JT, Diamond LE. Risk factors for lower limb injury in female team field and court sports: a systematic review, meta-analysis, and best evidence synthesis. Sports Med. 2021;51(4):759-776. doi:10.100 7/s40279-020-01410-9

13. Opar DA, Williams MD, Timmins RG, Hickey J, Duhig SI, Shield AJ. Eccentric hamstring strength and hamstring injury risk in Australian footballers. Med Sci Sports Exerc. 2015;47(4):857-865. doi:10.1249/MS $\underline{\mathrm{S} .0000000000000465}$

14. van Dyk N, Bahr R, Whiteley R, et al. Hamstring and quadriceps isokinetic strength deficits are weak risk factors for hamstring strain injuries: a 4-year cohort study. Am J Sports Med. 2016;44(7):1789-1795. doi:10.1177/0363546516632526

15. Burigo RL, Scoz RD, Alves BMO, et al. Concentric and eccentric isokinetic hamstring injury risk among 582 professional elite soccer players: a 10-years retrospective cohort study. BMJ Open Sport Exerc Med. 2020;6(1):e000868. doi:10.1136/bmjsem-2020-000868

16. Lee JWY, Mok KM, Chan HCK, Yung PSH, Chan KM. Eccentric hamstring strength deficit and poor hamstring-to-quadriceps ratio are risk factors for hamstring strain injury in football: A prospective study of 146 professional players. J Sci Med Sport. 2018;21(8):789-793. doi:10.1016/j.jsams.2017.11.017

17. Gabbe BJ, Bennell KL, Finch CF. Why are older Australian football players at greater risk of hamstring injury? J Sci Med Sport. 2006;9(4):327-333. doi:10.1016/j.jsams.2006.01.004

18. Witvrouw E, Danneels L, Asselman P, D’Have T, Cambier D. Muscle flexibility as a risk factor for developing muscle injuries in male professional soccer players: a prospective study. Am J Sports Med. 2003;31(1):41-46.

19. Bourne MN, Opar DA, Williams MD, Shield AJ. Eccentric knee flexor strength and risk of hamstring injuries in rugby union: a prospective study. Am J Sports Med. 2015;43(11):2663-2670. doi:10.1177/0363 $\underline{546515599633}$ 
20. Smith NA, Franettovich Smith MM, Bourne MN, Barrett RS, Hides JA. A prospective study of risk factors for hamstring injury in Australian Football League players. J Sports Sci. 2021:1-7. doi:10.1080/026 40414.2021.1875613

21. O’Connor S, McCaffrey N, Whyte EF, Fop M, Murphy B, Moran KA. Is poor hamstring flexibility a risk factor for hamstring injury in Gaelic games. $J$ Sport Rehabil. 2019;28(7):677-681. doi:10.1123/isr.201 $\underline{7-0304}$

22. Green B, Bourne MN, Pizzari T. Isokinetic strength assessment offers limited predictive validity for detecting risk of future hamstring strain in sport: a systematic review and meta-analysis. Br J Sports Med. 2018;52(5):329-336. doi:10.1136/bjsports-201 7-098101

23. Croisier JL, Forthomme B, Namurois MH, Vanderthommen M, Crielaard JM. Hamstring muscle strain recurrence and strength performance disorders. Am J Sports Med. 2002;30(2):199-203.

24. Croisier JL, Ganteaume S, Binet J, Genty M, Ferret JM. Strength imbalances and prevention of hamstring injury in professional soccer players: a prospective study. Am J Sports Med. 2008;36(8):1469-1475. doi:1 $\underline{0.1177 / 0363546508316764}$

25. Maniar N, Shield AJ, Williams MD, Timmins RG, Opar DA. Hamstring strength and flexibility after hamstring strain injury: a systematic review and meta-analysis. Br J Sports Med. 2016;50(15):909-920. doi:10.1136/bjsports-2015-095311

26. Hewett TE, Myer GD, Ford KR. Decrease in neuromuscular control about the knee with maturation in female athletes. J Bone Joint Surg Am. 2004;86-A(8):1601-1608.

27. Marshall PW, Siegler JC. Lower hamstring extensibility in men compared to women is explained by differences in stretch tolerance. BMC Musculoskelet Disord. 2014;15:223. doi:10.1186/1471-2474-15-223

28. Habets B, Staal JB, Tijssen M, van Cingel R. Intrarater reliability of the Humac NORM isokinetic dynamometer for strength measurements of the knee and shoulder muscles. BMC Res Notes. 2018;11(1):15. doi:10.1186/s13104-018-3128-9

29. Schneider-Kolsky ME, Hoving JL, Warren P, Connell DA. A comparison between clinical assessment and magnetic resonance imaging of acute hamstring injuries. Am J Sports Med. 2006;34(6):1008-1015. doi:10.1177/036354650528383 $\underline{5}$
30. Harvey D. Assessment of the flexibility of elite athletes using the modified Thomas test. Br J Sports Med. 1998;32(1):68-70.

31. Lephart SM, Abt JP, Ferris CM, et al.

Neuromuscular and biomechanical characteristic changes in high school athletes: a plyometric versus basic resistance program. Br J Sports Med. 2005;39(12):932-938.

32. Hewett TE, Stroupe AL, Nance TA, Noyes FR. Plyometric training in female athletes: Decreased impact forces and increased hamstring torques. Am J Sports Med. 1996;24(6):765-773.

33. Hewett TE, Myer GD, Zazulak BT. Hamstrings to quadriceps peak torque ratios diverge between sexes with increasing isokinetic angular velocity. J Sci Med Sport. 2008;11(5):452-459.

34. Aagaard P, Simonsen EB, Magnusson SP, Larsson B, Dyhre-Poulsen P. A new concept for isokinetic hamstring: quadriceps muscle strength ratio. Am J Sports Med. 1998;26(2):231-237.

35. Portney LG, Watkins MP. Foundations of Clinical Research. Upper Saddle River, NJ: Prentice Hall Inc.; 2000.

36. Orchard J, Marsden J, Lord S, Garlick D. Preseason hamstring muscle weakness associated with hamstring muscle injury in Australian footballers. Am J Sports Med. 1997;25(1):81-85.

37. van Dyk N, Behan FP, Whiteley R. Including the Nordic hamstring exercise in injury prevention programmes halves the rate of hamstring injuries: a systematic review and meta-analysis of 8459 athletes. Br J Sports Med. 2019;53(21):1362-1370. doi:10.1136/ bjsports-2018-100045

38. Vatovec R, Kozinc Z, Sarabon N. Exercise interventions to prevent hamstring injuries in athletes: A systematic review and meta-analysis. Eur J Sport Sci. 2020;20(7):992-1004. doi:10.1080/1746139 1.2019 .1689300

39. Al Attar WSA, Soomro N, Sinclair PJ, Pappas E, Sanders RH. Effect of injury prevention programs that include the Nordic hamstring exercise on hamstring injury rates in soccer players: a systematic review and meta-analysis. Sports Med. 2017;47(5):907-916. doi:1 0.1007/s40279-016-0638-2

40. Drury B, Ratel S, Clark CCT, Fernandes JFT, Moran J, Behm DG. Eccentric resistance training in youth: perspectives for long-term athletic development. J Funct Morphol Kinesiol. 2019;4(4). doi:10.3390/ifmk40 $\underline{40070}$ 
41. Lorenz D, Reiman M. The role and implementation of eccentric training in athletic rehabilitation: tendinopathy, hamstring strains, and acl reconstruction. Int J Sports Phys Ther.

$2011 ; 6(1): 27-44$.

42. Youdas JW, Krause DA, Hollman JH, Harmsen WS, Laskowski E. The influence of gender and age on hamstring muscle length in healthy adults. J Orthop Sports Phys Ther. 2005;35(4):246-252. doi:10.2519/jos pt.2005.35.4.246

43. Quatman CE, Ford KR, Myer GD, Paterno MV, Hewett TE. The effects of gender and pubertal status on generalized joint laxity in young athletes. J Sci Med Sport. 2008;11(3):257-263. doi:10.1016/j.jsams.2007.0 $\underline{5.005}$

44. Gabbe BJ, Finch CF, Wajswelner H, Bennell KL. Predictors of lower extremity injuries at the community level of Australian football. Clin J Sport Med. 2004;14(2):56-63.
45. Rogan S, Wust D, Schwitter T, Schmidtbleicher D. Static stretching of the hamstring muscle for injury prevention in football codes: a systematic review. Asian J Sports Med. 2013;4(1):1-9.

46. Quatman-Yates CC, Myer GD, Ford KR, Hewett TE. A longitudinal evaluation of maturational effects on lower extremity strength in female adolescent athletes. Pediatr Phys Ther. 2013;25(3):271-276. doi:1 0.1097/PEP.0b013e31828e1e9d

47. Biro FM, Lucky AW, Huster GA, Morrison JA. Pubertal staging in boys. J Pediatr. 1995;127(1):100-102.

48. Biro FM, McMahon RP, Striegel-Moore R, et al. Impact of timing of pubertal maturation on growth in black and white female adolescents: The National Heart, Lung, and Blood Institute Growth and Health Study. J Pediatr. 2001;138(5):636-643. doi:10.1067/mp d.2001.114476

49. Marshall WA, Tanner JM. Variations in the pattern of pubertal changes in boys. Arch Dis Child. 1970;45(239):13-23. 